\title{
Estimating the slip resistance from spherical nanoindentation and orientation measurements in polycrystalline samples of cubic metals
}

\author{
Dipen K. Patel and Surya R. Kalidindi \\ Woodruff School of Mechanical Engineering, Georgia Institute of Technology, Atlanta, GA 30332, USA
}

\begin{abstract}
In this paper, we demonstrate the first application of a recently formulated two-step approach to the estimation of the average slip resistance in a cubic polycrystalline metal sample from a collection of spherical nanoindentation and lattice orientation measurements. In the first step, a crystal plasticity finite element model of the spherical nanoindentation experiment is developed, validated, and employed to capture the functional dependence of the indentation yield strength on the crystal lattice orientation at the indentation site. This functional dependence is captured in a compact representation using surface spherical harmonics (SSH) functions. In the second step, measured values of the indentation yield strength and the crystal lattice orientations at the indentation site from a polycrystalline sample are fit to the function established in the first step to provide a robust estimate for the value of the average slip resistance in the sample. The validity of this approach is demonstrated for cast and annealed Fe-3\% Si using measurements published in prior literature.
\end{abstract}

Keywords: Spherical nanoindentation; Crystal plasticity; Initial slip resistance; Generalized Spherical Harmonics; Finite element models 


\section{Introduction}

Successful deployment of polycrystalline metals in demanding engineering applications requires a detailed understanding of their microscale deformation behavior. The deformation behavior of these materials is characterized and controlled by dislocation slip at the level of individual grains (i.e., crystals) in the polycrystalline sample. Accurate characterization of the slip resistance and other slip hardening parameters (Asgari et al., 1997; Beyerlein and Tomé, 2007; Bhattacharyya et al., 2001; Duchêne et al., 2007; Gambin and Barlat, 1997; Kalidindi et al., 2004; Kalidindi et al., 1992; Knezevic et al., 2010; Pathak et al., 2009; Sarma and Dawson, 1996; Vachhani et al., 2016) is essential to determining the macroscopic constitutive response of a polycrystalline aggregate subjected to finite plastic deformation.

Considerable attention has been paid to measure or estimate the slip resistance values and the slip hardening parameters from polycrystalline samples. A common practice in literature has been to extract these values by calibrating the polycrystalline deformation experiments with their corresponding simulations. For instance, the slip resistance values are estimated by calibrating the predicted macroscopic yield for different textures to the measured yield in multiple loading conditions (Barlat et al., 1997; Bron and Besson, 2004; Bronkhorst et al., 1991; Kalidindi and Anand, 1992; Kalidindi et al., 1992). Such approaches necessarily employ a homogenization scheme to relate the single crystal slip parameters to the effective polycrystalline response. Examples of such homogenization schemes range from the simple Taylor-type models (Taylor, 1934, 1938) to self-consistent models (Lebensohn and Tomé, 1993; Segurado et al., 2012; Tomé et al., 2002; Tomé et al., 2001) to micromechanical crystal plasticity finite element models (CPFEM) (Asaro and Needleman, 1985; Bachu and Kalidindi, 1998; Becker, 1991; Delannay et al., 2009; Kalidindi et al., 1992; Tikhovskiy et al., 2007). Consequently, the slip parameters 
extracted using these approaches are meant to be used exclusively with the specific homogenization schemes employed in estimating them in the first place. In other words, the calibrated slip parameters extracted in these approaches reflect an inherent bias introduced by the use of the homogenization model. Furthermore, since each test (combination of each texture and loading condition imposed on the sample) constitutes one data point, one typically needs to expend a substantial amount of time and effort to generate a sufficiently large dataset that provides robust values of the slip parameters of interest.

Alternately, it is possible to grow and test single crystals of many metals to estimate the values of the slip resistance. Such single-crystal experiments are effort and time intensive, as they require carefully controlled growth of preferentially oriented grains, their characterization via electron backscatter diffraction (EBSD) method, and the preparation of oriented test samples excised from these crystals. In practice, it is not always possible to produce sufficiently large volumes of the microscale constituents of interest in their pure form to allow the application of standardized mechanical testing methods (such as compression and tensile testing). Another approach explored in literature involves the compressive testing of micro-pillars fabricated using focused ion-beam (FIB) in a scanning electron microscope (SEM) (Li et al., 2011; Shan et al., 2008; Uchic et al., 2004). All of the techniques described above generally demand effort intensive sample preparation protocols and highly specialized test equipment.

Among the different experimental techniques explored thus far in literature, instrumented indentation (Doerner and Nix, 1986; Oliver and Pharr, 1992; Oliver and Pharr, 2004) exhibits tremendous potential as a low cost, high throughput, approach because of its capability to probe quickly multiple local volumes in a small sample. Indentation has been applied to study 
deformation behavior in a wide variety of material systems ranging from metals (Hannula et al., 1985; Mayo and Nix, 1988), polymers (Anand and Ames, 2006), and composites (Chen et al., 2010). Advances in instrumented indentation has facilitated studies of dislocation source activation (Nair et al., 2008; Zbib and Bahr, 2007), mechanical characterization of grain boundaries (Vachhani et al., 2016), slip lines in Ni-based single crystal supperalloy (Sabnis et al., 2012; Sabnis et al., 2013), and geometrically necessary dislocations produced by gradients of slip (Dahlberg et al., 2014). Indentation techniques have also been employed successfully to characterize the local mechanical responses in individual grains in a polycrystalline sample (Kalidindi and Pathak, 2008; Pathak, 2009; Pathak and Kalidindi, 2015; Pathak et al., 2008; Vachhani et al., 2016) (i.e., at length scales significantly lower than typical grain sizes). Although one can measure directly the local response at selected locations in a sample, one still needs a sophisticated strategy to extract the values of the slip parameters of interest from the measured load-displacement curves. The general approach (Dao et al., 2001; Kucharski and Mróz, 2001; Liu et al., 2008; Zaafarani et al., 2006) for addressing this challenge has been to employ optimization strategies that minimize a suitably defined error between the measurements (typically the load-displacement curves) and the corresponding predictions from a finite element (FE) simulation of the indentation experiment, by fine-tuning the model parameters of interest (such as slip resistances). Examples of such efforts have included the extraction of the average slip resistance value for single crystal copper (Liu et al., 2008; Zaafarani et al., 2006) and the hardening rate for low-alloy steels (Dao et al., 2001; Kucharski and Mróz, 2001). Since the entire elastic-plastic transition occurs over a very short regime in the indentation load-displacement curve (Kalidindi and Pathak, 2008; Pathak and Kalidindi, 2015), it is generally very difficult to identify this regime precisely on the measured load-displacement curve. Consequently, values of 
the slip parameters estimated using such approaches are generally not robust (i.e., they are very sensitive to small changes in the protocols employed). Indeed, it is much more practical and insightful to compare the indentation stress-strain curves instead of the load-displacement curves in calibrating the material constitutive laws (Donohue et al., 2012; Patel and Kalidindi, 2016). In this regard, the recently developed spherical nanoindentation data analysis protocols have shown tremendous potential (Kalidindi and Pathak, 2008; Pathak and Kalidindi, 2015). These new data analysis protocols convert the load-displacement data obtained during spherical nanoindentation into a meaningful indentation stress-strain (ISS) curve (details discussed later), which exhibits a clear initial elastic regime followed by an elastic-plastic transition (Kalidindi and Pathak, 2008; Pathak, 2009; Pathak et al., 2008; Pathak et al., 2009).

As a specific example that is highly relevant to the present work, the extraction and use of ISS curves has enabled the establishment of a robust correlation between indentation and uniaxial tests for materials exhibiting isotropic plastic response described by $\mathbf{J}_{2}$-flow theory (Hill, 1948). This was accomplished using a two-dimensional FE model of spherical indentation as a surrogate for the actual experiment. This is because the finite element model circumvents many of the uncertainties faced in the actual experiment (e.g., exact geometries of indenter and sample surfaces, friction between indenter and sample surface) offering opportunities for critical validation and demonstration of the data analyses protocols. This approach produced very simple scaling factors for the stress, elastic strain, and the plastic strain, which produced an excellent correlation between ISS curves and the conventional uniaxial stress-strain curves (Patel and Kalidindi, 2016). 
The present work builds on protocols described and applied previously by our research group (Patel et al., 2014) to extract single crystal anisotropic elastic stiffness constants for a cubic metal using spherical indentation measurements obtained on selected grains in a given polycrystalline material. A two-step protocol was developed and employed for this problem. In the first step, a three-dimensional finite element model of the spherical indentation was developed to capture the functional dependence of the elastic indentation modulus on the three cubic elastic stiffness parameters (usually denoted as $\mathrm{C}_{11}, \mathrm{C}_{12}$, and $\mathrm{C}_{44}$ ) and the crystal lattice orientation at the indentation site. Spherical surface harmonics (SSH) functions were utilized to capture the functional dependence on the orientation space, while Legendre polynomial bases were used to capture the functional dependencies on the three elastic stiffness parameters (over selected ranges of these parameters). It was shown that this strategy produces a compact and robust description of the function of interest. In the second step, the unknown single crystal elastic stiffness parameters for a given sample material were estimated by minimizing the differences between the predicted indentation moduli from the functional representation established in the first step to the corresponding indentation measurements made on differently oriented crystals. It was shown that the two-step protocol predicted the elastic stiffness parameters of cast and annealed $\mathrm{Fe}-3 \% \mathrm{Si}$ within a 5\% error margin.

The main objective of this work is to extend the methodologies developed in our previous work (Patel et al., 2014) to estimate the average slip resistance in a given polycrystalline sample of a cubic metal based on an ensemble of single crystal indentation measurements acquired on differently oriented grains in the sample. Crystal plasticity finite element (CPFEM) simulations of spherical indentation are ideally suited to extract a suitably formulated function for the first step in the two-step procedure described above. The computational cost associated with 
implementing high mesh density regions under the indenter coupled with the high cost of crystal plasticity constitutive calculations involved in the multiple loading-unloading cycles needed to establish the ISS curves, presents a major challenge for the present work. In this study, we have restricted our attention to cubic (i.e., fcc and bcc) metals. This allows us to consider a single value of an average slip resistance for all the slip systems in all grains in the given polycrystalline sample. This simplification is reasonable for cubic metals. The foundation presented here will allow future extensions to more sophisticated treatment of different slip resistances for different families of slip systems (e.g., hcp metals). The viability of these new protocols is demonstrated in this paper through an application to an annealed $\mathrm{Fe}-3 \% \mathrm{Si}$ polycrystalline sample, for which indentation measurements were previously reported (Pathak et al., 2009).

\section{Background}

\subsection{Spherical Indentation Stress-Strain (ISS) Curves}

The predicted and measured load-displacement data from indentation are best compared in the form of ISS curves, because such a comparison allows one to follow systematically the material response through the elastic regime, the elastic-plastic transition, and the plastic regime. The protocols involved in converting load-displacement data to indentation stress-strain (ISS) curves are based on the following set of equations (Kalidindi and Pathak, 2008):

$$
\sigma_{\text {ind }}=\frac{P}{\pi a^{2}}, \quad \varepsilon_{\text {ind }}=\frac{4}{3 \pi} \frac{h_{t}}{a} \approx \frac{h_{t}}{2.4 a}
$$


In Eq. (1), $\sigma_{\text {ind }}, \varepsilon_{\text {ind }}, P$, and $h_{t}$ denote an effective indentation stress, an effective indentation strain, the indentation load, and the total indentation displacement, respectively, while $a$ is the contact radius. In the linear elastic regime (i.e., $h_{t}=h_{e}$, where $h_{e}$ is the elastic indentation displacement), $\sigma_{\text {ind }}$ and $\varepsilon_{\text {ind }}$ demonstrate a linear relationship that is fully consistent with Hertz's theory (Hertz, 1896). Beyond the elastic regime, definitions in Eq. (1) have resulted in meaningful ISS curves that look similar to the stress-strain curves obtained from conventional bulk testing protocols (Donohue et al., 2012; Patel et al., 2014; Pathak, 2009; Pathak and Kalidindi, 2015; Pathak et al., 2009). The central challenge in the use of the definitions provided in Eq. (1) lies in the accurate estimation of the contact radius, $a$. The most robust approach to date for addressing this challenge has been to measure the unloading stiffness, $S$, and then estimate the contact radius using Hertz's theory (Hertz, 1896), which can be summarized in the following equations:

$$
\begin{aligned}
& P=\frac{4}{3} E_{e f f} R_{e f f}^{\frac{1}{2}} h_{e}^{\frac{3}{2}}, \quad \frac{1}{E_{e f f}}=\frac{1-v_{s}^{2}}{E_{s}}+\frac{1-v_{i}^{2}}{E_{i}} \\
& a=\frac{S}{2 E_{e f f}}, \quad \frac{1}{R_{e f f}}=\frac{1}{R_{s}}+\frac{1}{R_{i}}
\end{aligned}
$$

Eq. (2) is only applicable for the case of frictionless contact between two linear elastic, isotropic, quadratic solid surfaces, namely the indenter and the sample, whose geometry and properties are indicated by variables with subscripts $i$ and $s$, respectively. The subscript eff is used to denote an effective attribute of the combined indenter-sample system. $E, v$, and $R$ denote the Young's modulus, the Poisson's ratio, and the radius, respectively. Although the theory described above was originally intended for an elastically isotropic material, it has been shown subsequently that 
the same approach can be extended to elastically anisotropic materials with the interpretation that the modulus extracted represents an "effective" value in the indentation direction (Gao and Pharr, 2007; Patel et al., 2014; Pathak et al., 2009; Swadener and Pharr, 2001; Vlassak et al., 2003; Vlassak and Nix, 1993, 1994; Willis, 1966).

Since Hertz's theory is only applicable to elastic indentation, it can only be applied on the initial elastic loading segment and any elastic unloading segment imposed after some plastic strain has been introduced in the sample during the indentation. Consequently, it is essential to superimpose multiple unloading segments on the monotonic loading segment typically employed in the indentation test. In the modern nanoindenters, this is accomplished with the continuous stiffness measurement (CSM) option (Oliver and Pharr, 1992; Pethicai et al., 1983; Pharr, 1998). In the simulations, to be consistent with the experimental protocols, one has to introduce unloading segments (see Fig. 1) explicitly to estimate a value of the contact radius that is consistent with Hertz's theory (Hertz, 1896). Since each unloading segment produces one data point on the ISS curve and adds a substantial computational cost to the overall simulation, considerable thought should be afforded in designing the precise loading-unloading history in the indentation simulation in order to recover a meaningful ISS curve. 
a) Continuous Stiffness Measurements

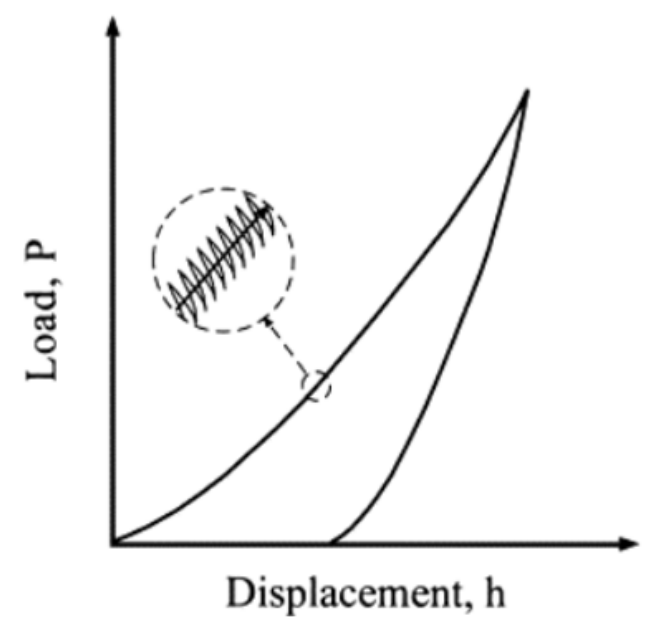

b) Load-unload in FE simulations

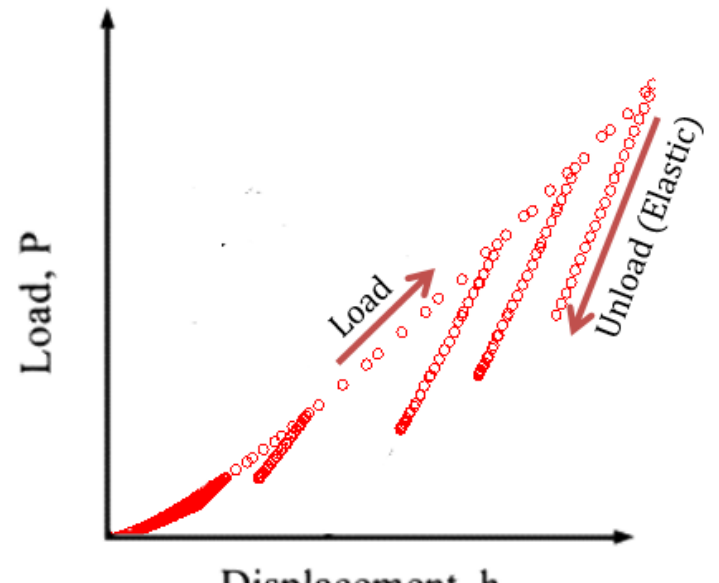

Displacement, $\mathrm{h}$

Figure 1. a) Schematic of CSM signal superimposed on the loading cycle in nanoindentation experiment. b) Schematic of load-unload cycles to replicate CSM signal in finite element simulations.

\subsection{Extracting Crystal-Level Properties from Indentation Measurements}

Nanoindentation on polycrystalline samples (Kalidindi and Pathak, 2008; Kalidindi and Vachhani, 2014; Liu et al., 2008; Pathak, 2009; Pathak and Kalidindi, 2015; Pathak et al., 2008; Pathak et al., 2009; Vachhani et al., 2016; Vachhani and Kalidindi, 2015; Zaafarani et al., 2006) is typically used to evaluate local mechanical responses at length scales that are significantly smaller than the grain size. In other words, the indentation zones are in most cases are single crystal regions. While measuring the ISS curves in single crystals regions of different lattice orientations within a polycrystalline sample is now a relatively straightforward task, relating and extracting reliable crystal level properties from these ISS curves is quite challenging. 
In recent work, we have described a two-step protocol to extract grain-scale elastic stiffness constants from the ISS curves. In the first step of the two-step process, a functional dependence of the effective sample indentation modulus, $E_{e f f}$, over the entire orientation space for a large range of the (unknown) crystal elastic stiffness parameters is captured in the form of highly efficient spectral representations (Patel et al., 2014) expressed as

$E_{e f f}\left(g, C_{11}, C_{12}, C_{44}\right)=\sum_{l=0}^{\infty} \sum_{m=1}^{M(l)} \sum_{q, r, s=0}^{\infty} A_{l}^{m q r s} K_{l}^{m}(g) P_{q}\left(C_{11}\right) P_{r}\left(C_{12}\right) P_{s}\left(C_{44}\right)$

where $K_{l}^{m}$ and $P_{x}$ are spherical surface harmonics (SSH) and Legendre polynomials, respectively. These representations are in many ways similar to those used successfully in prior work for establishing microstructure-property linkages (Adams et al., 2013; Proust and Kalidindi, 2006; Yabansu and Kalidindi, 2015; Yabansu et al., 2014). The data needed for this step is collected by executing a suitable finite element (FE) model of the indentation multiple times (corresponding to different combinatorial selections of the lattice orientation and the single crystal elastic constants of the sample material). In the second step, a methodology is established to extract the unknown single crystal material parameters for a selected phase in polycrystalline materials by minimizing the difference between the measured indentation moduli on differently oriented grains, $\tilde{E}_{\text {eff }}(g)$ and the spectral representation established in step one, $E_{e f f}\left(g, C_{11}, C_{12}, C_{44}\right)$ via regression analysis. The two-step protocol has been successfully implemented to estimate the single crystal elastic constants $\left(\mathrm{C}_{11}, \mathrm{C}_{12}\right.$, and $\left.\mathrm{C}_{44}\right)$ of cast and annealed Fe3\%Si polycrystalline material (Patel et al., 2014). 
As mentioned earlier, the central goal of this study is to extend these recent developments to estimating the effective slip resistance, $s$, in cubic polycrystalline samples. Assuming that this value is uniform on all the slips systems in the indentation zone, the desired function (analogous to Eq. (3)) can be expressed as

$\tilde{Y}_{\text {ind }}(g)=\frac{Y_{\text {ind }}(g)}{s}=\sum_{l=0}^{\infty} \sum_{m=1}^{M(l)} B_{l}^{m} K_{l}^{m}(g)$

where $Y_{\text {ind }}$ is the indentation yield strength, and $\tilde{Y}_{\text {ind }}(g)$ is a normalized indentation yield strength. Note that these two definitions of the indentation yield strength are linearly related through the slip resistance. In many ways, it should actually be much easier to establish the function described in Eq. (4) compared to what was done in the previous study (Patel et al., 2014) to establish Eq. (3). However, the central challenges are: (i) the CPFEM simulations needed to establish the SSH coefficients, $B_{l}^{m}$, are computationally much more demanding, and (ii) the number of coefficients needed are expected to be significantly higher (plastic properties are known to require many more terms compared to elastic properties in the Fourier representations (Adams et al., 2013; Fullwood et al., 2010).

\section{Crystal Plasticity Finite Element Simulation of Spherical Nanoindentation}

As described in the previous section, the first step in our protocol requires the generation of a dataset that can be used to estimate the Fourier coefficients in Eq. (4). It is important to build a computationally efficient crystal plasticity-based FE model of the spherical indentation for this purpose. Since we intend to use this model to simulate indentations over a broad range of crystal orientations, the computational efficiency of the model is of utmost importance. In this work, we 
have employed a previously established and validated model (Kalidindi et al., 1992) as a UMAT (user material subroutine) in ABAQUS (ABAQUS, 2014) to simulate the spherical indentation test. A complete description of this model along with the implementation details have already been published in prior literature (Bachu and Kalidindi, 1998; Bhattacharyya et al., 2001; Kalidindi and Anand, 1993, 1994; Kalidindi et al., 2004; Kalidindi et al., 2009; Kalidindi and Schoenfeld, 2000). Below, we focus mainly on the details of the FE model for spherical indentation.

Because the sample in the indentation simulations is likely to be assigned an arbitrary lattice orientation, we cannot take advantage of any symmetry in the indenter geometry (the sample response is fully anisotropic). Consequently, it is necessary to develop a complete threedimensional FE model of the indenter-sample system for the present study. In general, a fine mesh density is needed to capture reliably the evolving elastic-plastic response under the indenter. However, it is important to note that increasing the number of finite elements in the mesh increases the computational cost per simulation significantly. A large number of different meshing schemes were explored in this study before settling on the final model.

The FE model developed and employed for this study is shown in Fig. 2a and comprises of two three-dimensional solids: (i) an elastic-plastic deformable sample with an initially flat surface, and (ii) a rigid hemi-spherical indenter of radius $13.5 \mu \mathrm{m}$ (typical size of the indenter in prior experimental studies (Kalidindi and Pathak, 2008; Pathak, 2009; Pathak et al., 2009). Eightnoded, three-dimensional, continuum elements (C3D8 in ABAQUS (ABAQUS, 2014)) were used to mesh the deformable part. The size of the sample was selected as $10 \mu m X 10 \mu m X 10 \mu m$ to ensure that the stress-strain fields are well within the primary indentation zone size. There 
were a total of $17 \mathrm{~K}$ elements within the indentation zone, whose overall length scale was observed to be about $\sim 2.5 \mu \mathrm{m}$ from our simulations. The indentation zone has been discretized into seven regions as shown in Fig. 2a to permit the use of progressively higher mesh density as we approach the indentation zone directly below the indenter-sample contact surface. This partitioning allows highest mesh densities in the regions where steepest gradient in the stress (or strain) fields are expected in the sample. With this meshing scheme, the innermost region $(0.1 \mu m X 0.1 \mu m X 0.07 \mu m)$ has an element size of $(3.9 \mathrm{~nm} X 3.9 \mathrm{~nm} X 4.4 \mathrm{~nm})$. The aspect ratio of the element in the indentation zone was 1.8. The aspect ratio of the elements outside the indentation zone is larger, but these regions experience fairly minimal stresses and strains. A hard surface-to-surface, frictionless, contact was defined between the sample and indenter. A vertical displacement boundary condition was imposed on the indenter. The bottom surface of the sample was constrained along the z-direction (indentation direction). Encastre boundary condition is defined at the center of the bottom surface of the sample to arrest rigid body translations and rotations. The lateral surfaces of the sample material are kept free throughout the simulation. The displacement of the indenter and the total force applied on the sample are recorded at every time increment from the simulation. 


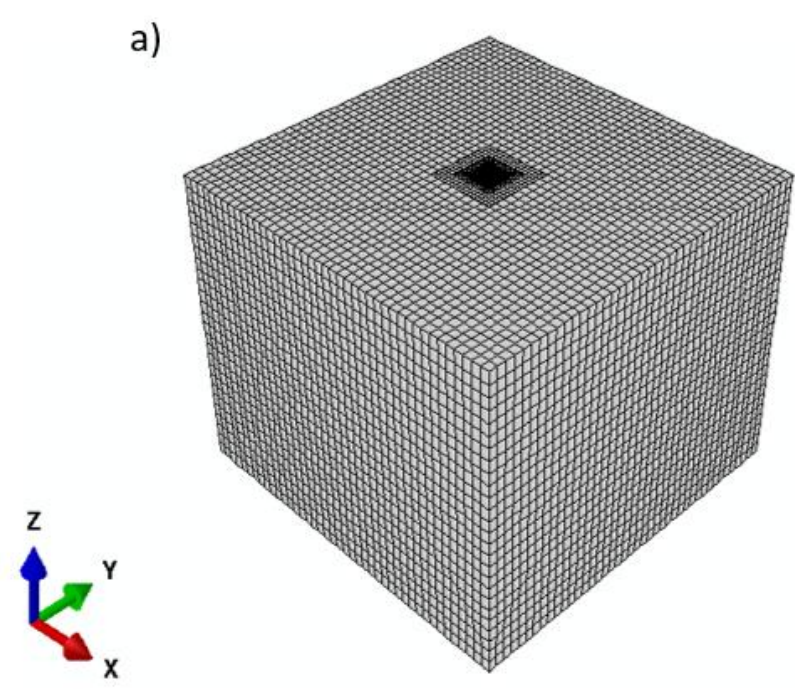

b)

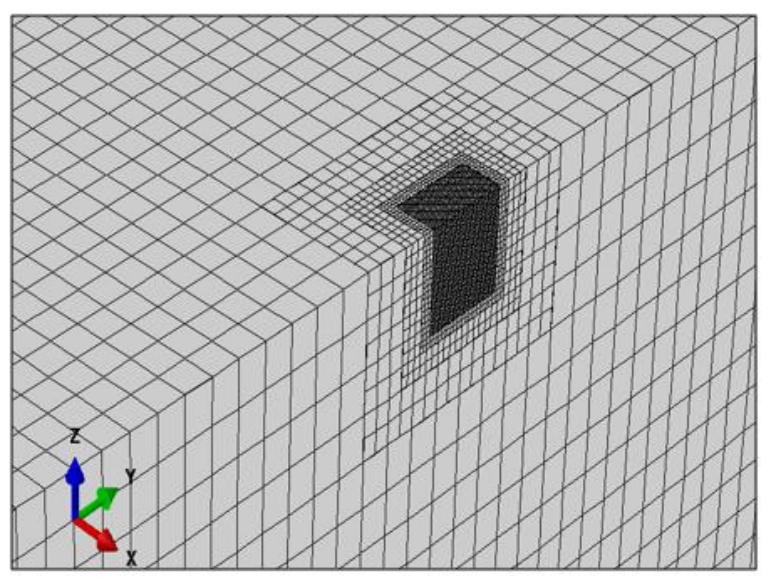

Figure 2. a) Finite element mesh of the sample in the spherical nanoindentation. b) Close-up view of a midsection through the indentation zone under the indenter tip.

The finite element model developed in this study was validated by comparing the indentation stress-strain curve for isotropic perfectly-plastic material constitutive behavior with the previously published result obtained using a 2-D FE model (see Fig 3). The FE mesh developed and utilized in this work enabled a relatively fast computation of the ISS curves using the crystal plasticity constitutive model. The final mesh employed in this work executed the multiple loading-unloading segments needed to recover the ISS curves in about 5 hours per simulation on an AMD "Bulldozer" 20 CPUs provided by Partnership for an Advanced Computing Environment (PACE) facility. 


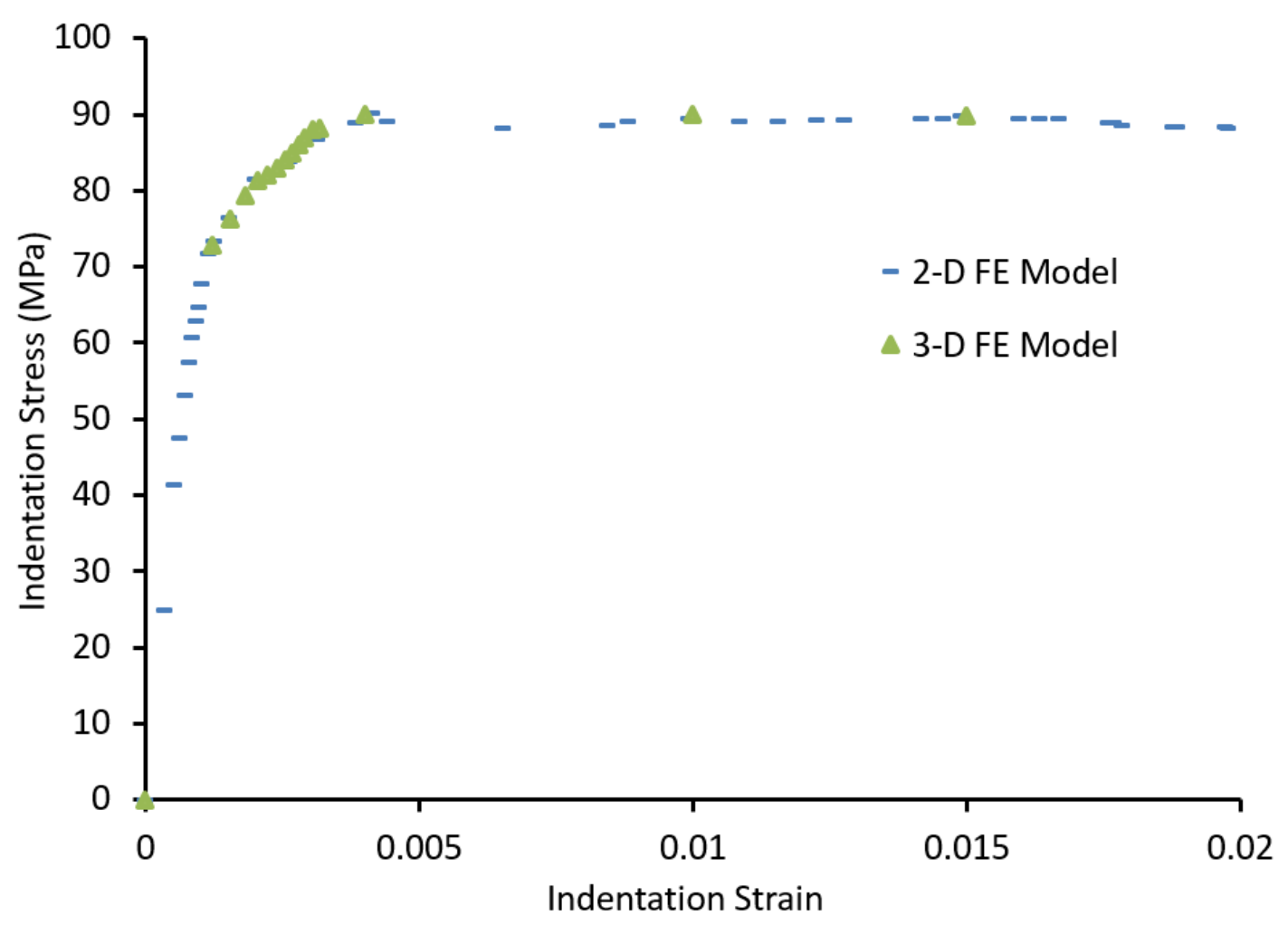

Figure 3. Comparison of the predicted ISS response from FE simulation against the corresponding prediction from previously published ISS curve using 2-D FE simulations of indentation for isotropic perfectly plastic response (Patel et al., 2016).

\section{Orientation Dependence of Indentation Yield in BCC Crystals}

As discussed earlier, our first goal in this work is to establish the Fourier coefficients, $B_{l}^{m}$, in Eq. (4). Our strategy will be to numerically evaluate $\tilde{Y}_{\text {ind }}(g)$ for a sufficiently large number of crystal orientations using the crystal plasticity FE model described in the previous section, and to use this data to estimate (or calibrate) the Fourier coefficients, $B_{l}^{m}$, using a linear regression method (Patel et al., 2014). The inputs to the crystal plasticity FE simulations of spherical indentation are the single crystal elastic stiffness constants, the crystal lattice orientation $(g)$, and arbitrarily chosen average initial slip resistance parameter, $s_{0}=8 \mathrm{MPa}$ for all 48 slip systems (Kocks et al., 2000). It is important to note that the sample is assumed to initially be a single crystal region (i.e., all elements are assigned the same initial crystal lattice orientation) and is 
assumed to be non-hardening (as this has no relevance to our goal of establishing the function defined in Eq. (4)). The crystal orientation is described by a set of three Bunge-Euler angles (Bunge, 1969) denoted as $\left(\varphi_{1}, \Phi, \varphi_{2}\right)$. Since, any arbitrary rotation of the sample about the axis of the spherical indenter does not influence the indentation response, the extracted ISS curves and the value of $Y_{i n d}$ only depend on two of the three Bunge-Euler angles, $\left(\Phi, \varphi_{2}\right)$. Considering the inherent crystal cubic symmetry and the transversely-isotropic sample symmetry, the crystal lattice orientations for different simulations were selected from the fundamental zone (FZ) defined by

$F Z=\left[\left(\Phi, \varphi_{2}\right) \mid \cos ^{-1}\left(\frac{\cos \varphi_{2}}{\sqrt{1+\cos ^{2} \varphi_{2}}}\right) \leq \Phi \leq \frac{\pi}{2}, \quad 0 \leq \varphi_{2} \leq \frac{\pi}{4}\right]$

For the present study, 36 crystal lattice orientations were selected from the FZ by discretizing the orientation space in a way that ensures that the bins have the same invariant measure (see Fig. 4; cf. (Fullwood et al., 2010)). The indentation yield strength is extracted from the ISS curve using a $0.2 \%$ offset indentation plastic strain.

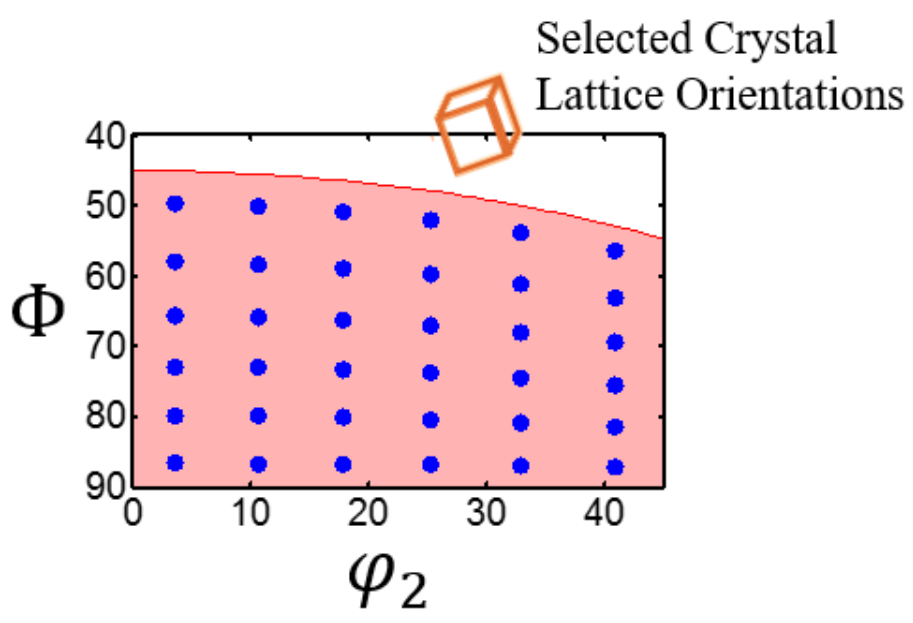

Figure 4. Distinct set of selected crystal lattice orientations spanning the cubic-transversely isotropic fundamental zone (highlighted area). 
An ordinary least square approach described in our previous work (Patel et al., 2014) has been used to calibrate the Fourier coefficients, $B_{l}^{m}$ (see Eq. (4)). Through repeated trials, it was found that truncating the Fourier representation at $l=6$ provided a good representation. This truncation level results in a total of 23 Fourier coefficients. The truncation error was observed to be less than 5\%. This is consistent with prior work on the microstructure-sensitive design (MSD) framework, where plastic properties such as tensile and shear yield strength showed similar error levels when truncated at $l=6$ (Proust and Kalidindi, 2006).

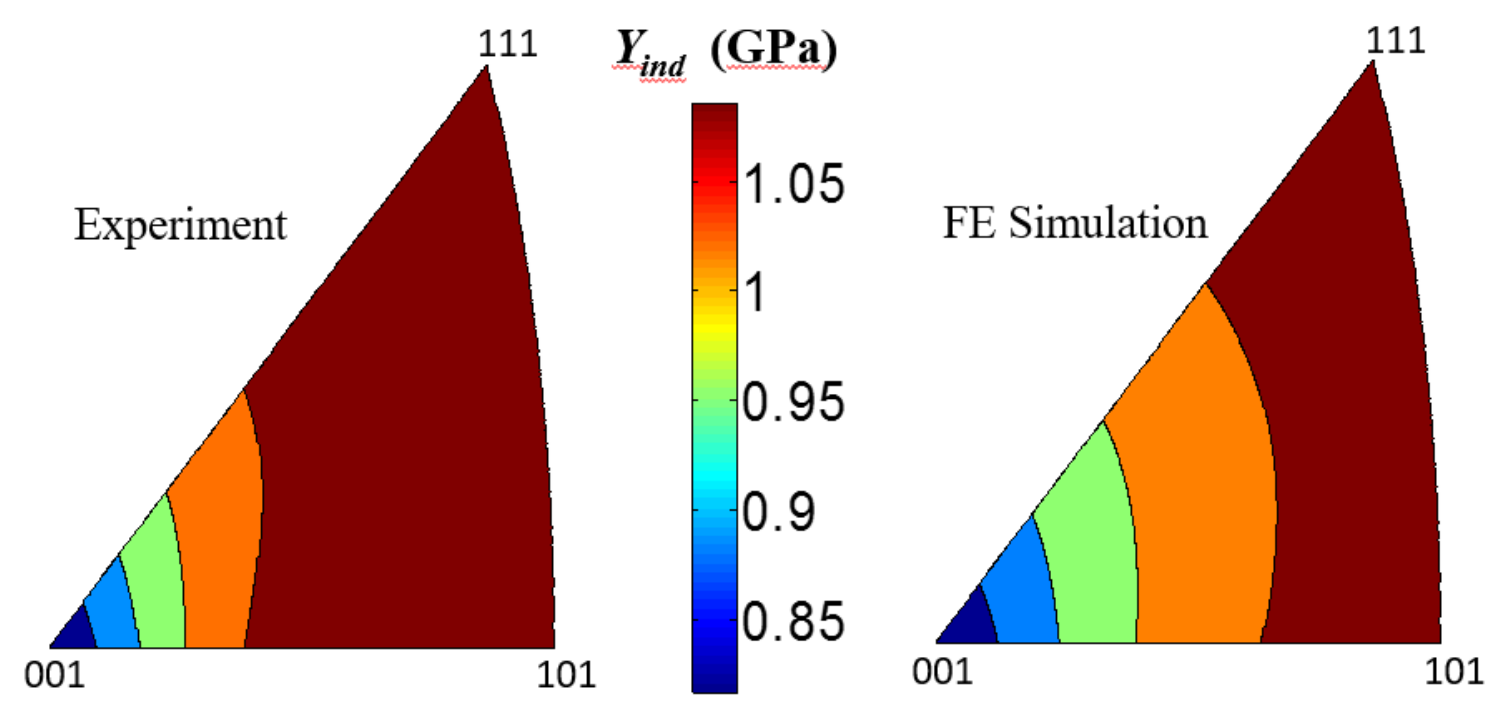

Figure 5. Inverse pole figure (IPF) map showing the surface contours of the variation in the indentation yield strength over the orientation space based on experimental observations and FE simulations.

As another validation, we present in Figure 5 a comparison between $\tilde{Y}_{i n d}(g)$ established in this work and the $Y_{\text {ind }}(g)$ measured experimentally in prior work on an cast and annealed Fe3\% Si BCC polycrystalline sample (Pathak et al., 2009). Note that it is not possible to compare directly the predicted and measured indentation yield strengths, since the value of the slip resistance for the sample used in the experiment is unknown. The good agreement in the shapes 
of the contours shown in Figure 5 suggests that we should be able to extract the value of the slip resistance simply by calibrating the experimental results to the FE predicted results. This is pursued next.

\section{Estimating the Initial Slip Resistance}

The main goal of this work is to develop and validate a robust methodology to extract the value of the local (i.e., length scales well below the typical grain size) slip resistance from a polycrystalline bcc sample. This will be accomplished by minimizing the difference between CPFEM predicted indentation yield values and the corresponding measured values on a selected set of grain orientations in the given polycrystalline sample. A suitable definition of the error for such a regression analyses can be expressed as

$\chi^{2}(s)=\sum_{j=1}^{N}\left[\frac{Y_{i n d}^{E x p}\left(g_{j}\right)-\left(s \tilde{Y}_{i n d}\left(g_{j}\right)\right)}{\sigma_{j}}\right]^{2}$

where $j$ enumerates the grain orientations where the measurements were made, $N$ is total number of grain orientations studied in the given sample, and $\sigma_{j}$ denotes the standard deviation for multiple measurements on a single grain (this allows us to account for potentially different levels of variance in the measurements in the differently oriented grains). The value of the slip resistance, $s$, can then be determined easily by minimizing the $\chi^{2}$ function (i.e., setting its derivative with $s$ to zero). This value can be computed as 


$$
s=\frac{\sum_{j=1}^{N} \frac{\tilde{Y}_{i n d}\left(g_{j}\right) Y_{i n d}^{E x p}\left(g_{j}\right)}{\sigma_{j}{ }^{2}}}{\sum_{j=1}^{N} \frac{\left.\tilde{Y}_{i n d}\left(g_{j}\right) \tilde{Y}_{i n d}\left(g_{j}\right)\right)}{\sigma_{j}{ }^{2}}} .
$$

It is possible to compute the variance on the estimated value of the slip resistance (as a measure of the uncertainty in the estimated value) as (Press, 2007)

$$
\sigma_{s}^{2}=\sum_{j=1}^{N} \sigma_{j}^{2} \frac{\partial s}{\left.\partial Y_{i n d}^{E x p}(g)\right|_{g=g_{j}}}=\frac{\sum_{j=1}^{N} \tilde{Y}_{i n d}\left(g_{j}\right)}{\sum_{k=1}^{N} \frac{\left.\tilde{Y}_{i n d}\left(g_{k}\right) \tilde{Y}_{i n d}\left(g_{k}\right)\right)}{\sigma_{k}{ }^{2}}}
$$

\section{Case study: Cast and Annealed Fe3\%Si Polycrystalline Sample}

The protocols described in the earlier sections will now be demonstrated on spherical indentations measurements reported previously (Pathak et al., 2009) on extremely large grains (of the order of few millimeters) in cast and annealed Fe-3\% Si polycrystalline steel sample. As pointed out by the authors of that study, the large grains significantly reduce the probability for the existence of a grain boundary just below the indentation surface or in the vicinity of the indentation site (selected in the interior parts of large grains). It is also noted that this previous study reported measurements on three different samples (only one is annealed and the other two are plastically deformed). In the present work, we are only estimating the slip resistance for the cast and annealed sample. For the convenience of the reader (and to avoid any confusion about what measurements were used in this study), the measurements utilized in this study are reproduced in Table 1. The regression techniques described in the previous section produced an estimated value (along with estimated variance) of the slip resistance as $155.4 \pm 3.5 \mathrm{MPa}$ for the cast and annealed $\mathrm{Fe}-3 \% \mathrm{Si}$ polycrystalline material. This estimated value lies within the range of 
values reported in prior literature for Fe-3\% Si using a variety of protocols. For example, a value of 146.12 MPa (Hull, 1963) was obtained from single crystals deformed in tension. A value of $161 \mathrm{MPa}$ (Klusemann et al., 2012; Orlans-Joliet et al., 1990) was obtained from calibrating the tensile test data on single crystals of $\mathrm{Fe}-3 \% \mathrm{Si}$ for different lattice orientations via crystal plasticity finite element analysis. Additionally, the effective slip resistance value was also estimated from measurements on polycrystalline samples of $\mathrm{Fe}-3 \% \mathrm{Si}$. These values fall within the range of values obtained from the single crystals studies. For instance, from tensile studies on fine $(20 \mu \mathrm{m})$ and coarse $(170 \mu \mathrm{m})$ grain sized Fe-3\%Si (Suits and Chalmers, 1961), it was deduced that the yielding is independent of the grain size and the value of slip resistance was estimated to be in the range of $130.42 \mathrm{MPa}$ and $157.9 \mathrm{MPa}$. Clearly, the values obtained in this study are in good agreement with those obtained in prior studies described above.

\begin{tabular}{|c|c|c|c|}
\hline \multicolumn{4}{|c|}{ As-cast Fe-3\%Si } \\
\hline & $\begin{array}{c}\text { Grain } \\
\text { No. }\end{array}$ & $\begin{array}{l}\text { Orientation } \\
\left(\varphi_{1}, \Phi, \varphi_{2}\right)\end{array}$ & $\begin{array}{c}\text { Experimental } \\
\boldsymbol{Y}_{\text {ind }}(\mathbf{G P a})\end{array}$ \\
\hline \multirow{4}{*}{$\begin{array}{l}\text { Near } \\
(111)\end{array}$} & 2 & $339.8,54.4,46.1$ & $1.13 \pm 0.04$ \\
\hline & 7 & $103.7,121.6,49.9$ & $1.12 \pm 0.02$ \\
\hline & 10 & $232.5,53.1,324.0$ & $1.12 \pm 0.16$ \\
\hline & 6 & $83.2,125.4,30.4$ & $1.10 \pm 0.02$ \\
\hline \multirow{3}{*}{$\begin{array}{l}\text { Near } \\
(101)\end{array}$} & 3 & $3.0,41.3,76.4$ & $1.09 \pm 0.04$ \\
\hline & 5 & $194.7,79.7,317$ & $1.07 \pm 0.01$ \\
\hline & 9 & $50.0,38.1,250.1$ & $1.06 \pm 0.02$ \\
\hline \multirow{4}{*}{$\begin{array}{l}\text { Near } \\
(001)\end{array}$} & 1 & $114.2,85,173.5$ & $0.85 \pm 0.04$ \\
\hline & 8 & $170.0,102.6,357.9$ & $0.91 \pm 0.06$ \\
\hline & 4 & $163.6,78.8,168$ & $0.93 \pm 0.04$ \\
\hline & 11 & $259.9,238.0,145.8$ & $1.0 \pm 0.06$ \\
\hline
\end{tabular}

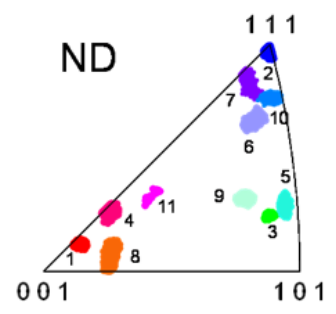

b)

Table 1. a) Measured values of the indentation yield, $Y_{\text {ind }}$, for eleven different orientations, and b) representation of the orientations in an inverse pole figure map (Pathak et al., 2009). 
The robustness of the estimated value of the slip resistance from the protocols described in this paper (i.e., sensitivity of the estimated value to the choices made in the estimation protocols) depends critically on the number and quality of the indentation yield measurements available, and the orientation space they cover. Note that indentation yield measurements were available from only 11 distinct grain orientations for this study. In general, a critical selection of the grain orientations for the indentation measurements is essential for a reliable estimation of the slip resistance value. Because of the regression method used in the approach described here for the estimation of the effective slip resistance, it is important to select orientations that produce robust estimates of the slip resistance.

In order to systematically evaluate the robustness of the estimated value of the slip resistance, one can subsample the measurements and repeat the entire estimation protocol. Let $N$ (in the present case study, $N=11$ ) denote the total number of grain orientations studied using indentation protocols described in this paper. If one were to decide to make indentation measurements on only $k$ grain orientations $(k \leq N)$, it would be possible to identify $\left\langle\begin{array}{l}N \\ k\end{array}\right\rangle=\frac{N !}{k !(N-k) !}$ distinct ensembles (i.e., subsamples) of potential datasets that might have been collected. As an example, if $k=1$, the number of distinct ensembles is 11 for the present case study. For $k=2$, the number of distinct ensembles is 55. A study of the estimated values of the slip resistance from each such ensemble will provide insights into how many grain orientations are needed to obtain reliable results. It will also provide some insights on how to select the grain orientations for the indentation measurements. 

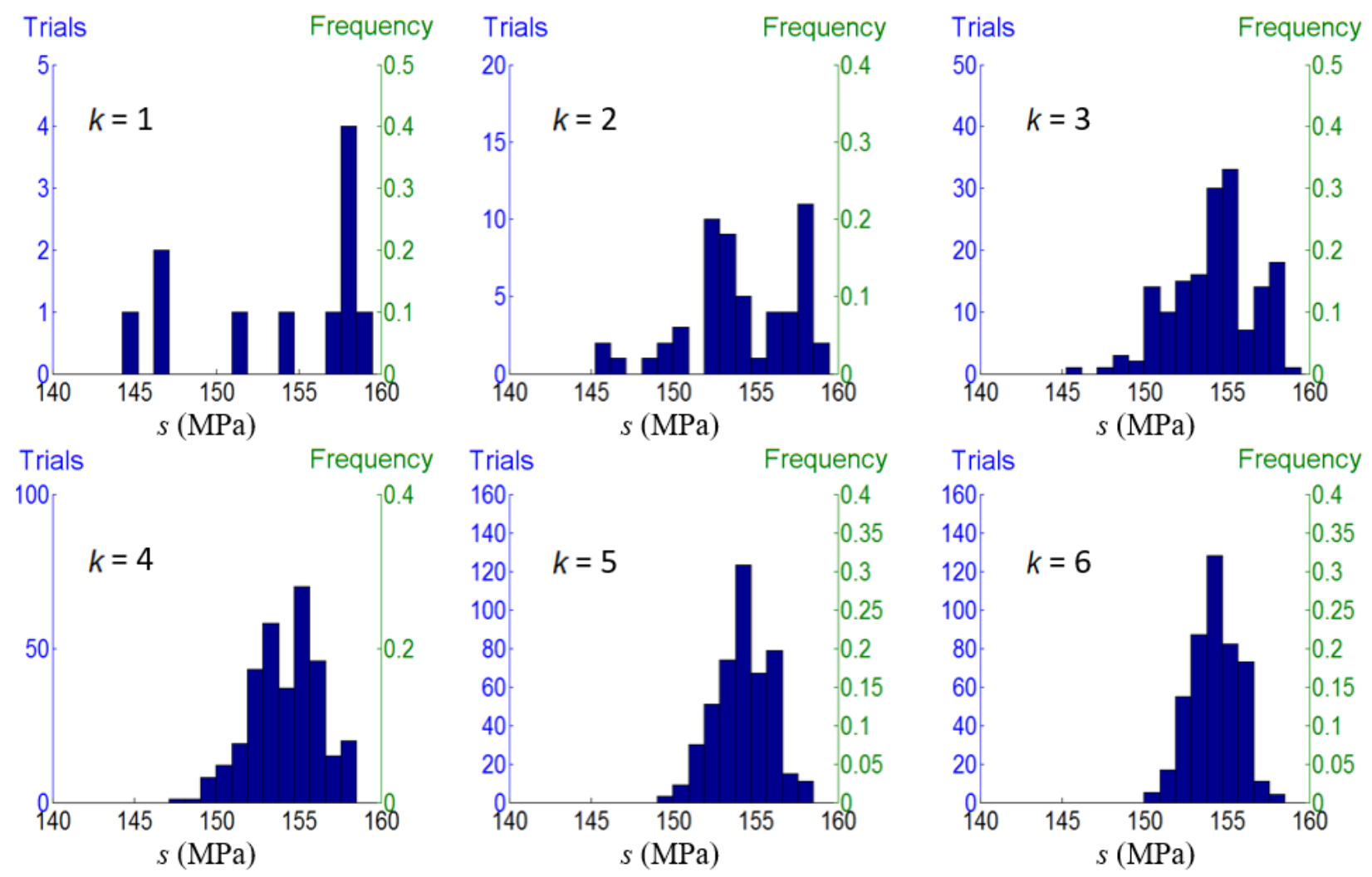

Figure 6. The frequency distribution of the estimated slip resistance values using various ensembles of subsamples.

The estimated values of the slip resistance for the different choices of $k$ from the ensembles created as described above are summarized in Figure 6 as frequency plots. As expected, the distribution of the estimated value of the slip resistance sharpens significantly with an increase in $k$. Interestingly, as stated earlier, the distribution of the estimated values is mostly within 155.4 $\mathrm{MPa} \pm 3.5 \mathrm{MPa}$ for the higher values of $k$ (see $k=6$ in Figure 6). One might look at the percentage of estimated values within $3 \%$ of the mean estimated value for each value of $k$ to determine how many grain orientations are needed to establish a reliable value of the slip resistance using the protocols described in this paper. This value increases from $72 \%$ for $k=1$, to $87 \%$ for $k=2$, to $93 \%$ for $k=3$, to $96 \%$ for $k=4$. This simple analysis suggests that there is a high likelihood of obtaining a reliable value of the slip resistance with indentation measurements 
in as few as four different grain orientations. Figure 7 shows the specific ensembles (i.e., selections of four grain orientations) that produced the closest estimates to the value of 155.4 MPa estimated in this study. From a review of these inverse pole figures, the following guidelines emerge for the selection of the grain orientations for the indentation measurements: (i) select grains with orientations close to each of the corners of the inverse pole figures, and (ii) distribute the orientations as uniformly as possible to cover the fundamental zone of the orientation space.
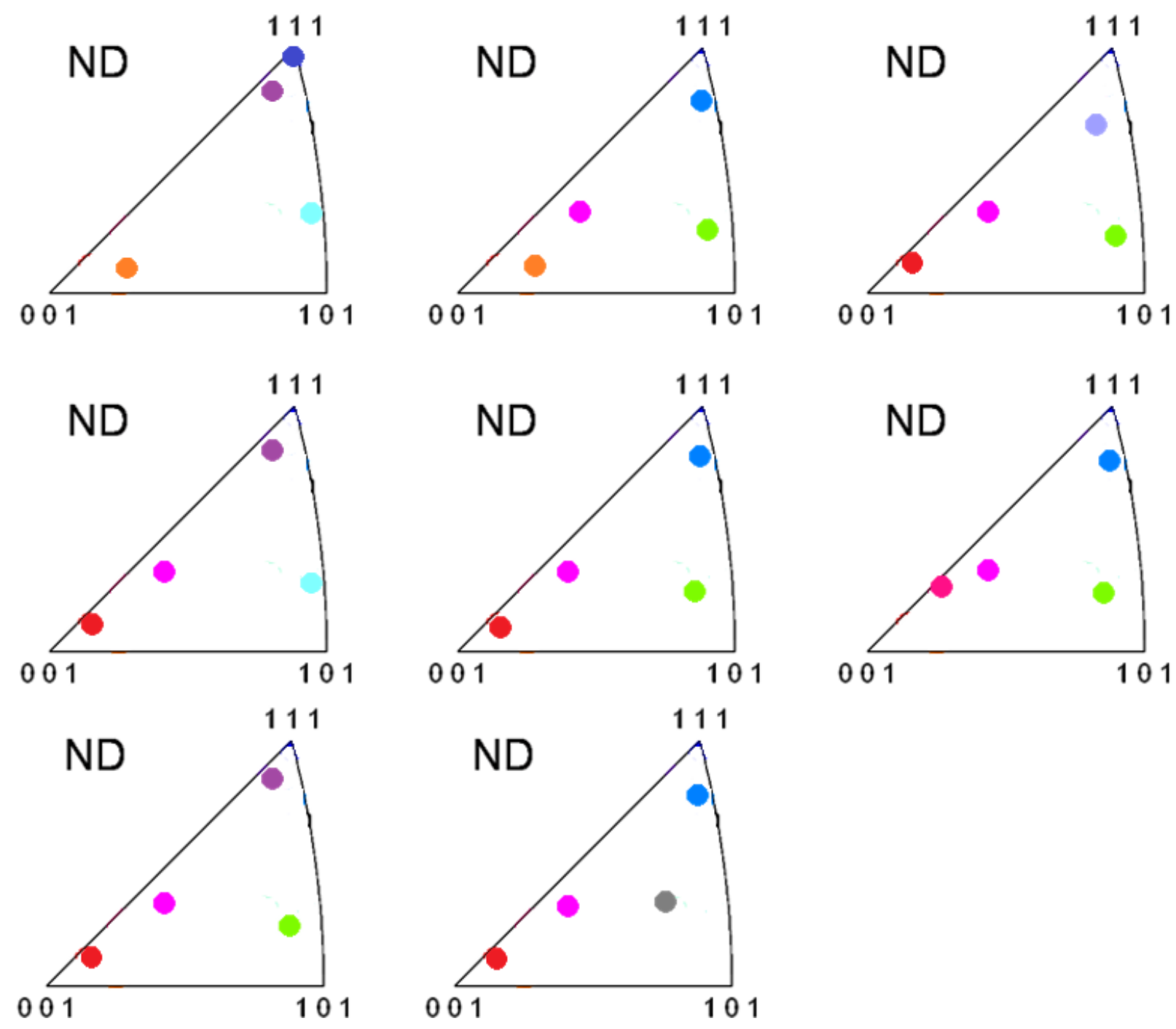

Figure 7. Ensembles from $k=4$ (four grain orientations selected randomly) that produced values closest to the estimated slip resistance of $155.4 \mathrm{MPa}$ from the entire collection of eleven grain orientations. 


\section{Conclusion}

A novel two-step protocol has been developed and demonstrated to provide robust estimates of the effective slip resistance from spherical nanoindentation and orientation measurements conducted on polycrystalline samples. The first step involved a substantial one-time computational effort to capture the functional dependence of the indentation yield on the lattice orientation for a selected material system. This step was accomplished in this study for bcc crystals by employing a CPFEM of spherical indentation. In the second step, the unknown value of the slip resistance was estimated through a simple regression technique. The accuracy and viability of the proposed approach were demonstrated for cast and annealed bcc polycrystalline $\mathrm{Fe}-3 \%$ Si sample. As already pointed out, the approach demonstrated in this paper is only likely to work for cubic metals, where it is reasonable to assume that the different slip systems have the same slip resistance. Considerable further development is needed to extend this approach to less symmetric metals (such as hexagonal metals) where it is important to account for the stark differences in the slip resistances of the different slip systems.

\section{Acknowledgements}

The authors gratefully acknowledge support from the U.S. Department of Energy, Office of Nuclear Engineering, Nuclear Engineering Enabling Technologies (DOE-NEET). 


\section{References}

ABAQUS, 2014. Simulia Providence.

Adams, B.L., Kalidindi, S., Fullwood, D.T., 2013. Microstructure-sensitive Design for Performance Optimization. Butterworth-Heinemann.

Anand, L., Ames, N.M., 2006. On modeling the micro-indentation response of an amorphous polymer. International Journal of Plasticity 22, 1123-1170.

Asaro, R.J., Needleman, A., 1985. Overview no. 42 Texture development and strain hardening in rate dependent polycrystals. Acta Metallurgica 33, 923-953.

Asgari, S., ElDanaf, E., Kalidindi, S.R., Doherty, R.D., 1997. Strain hardening regimes and microstructural evolution during large strain compression of low stacking fault energy fcc alloys that form deformation twins. Metallurgical and Materials Transactions a-Physical Metallurgy and Materials Science 28, 1781-1795.

Bachu, V., Kalidindi, S.R., 1998. On the accuracy of the predictions of texture evolution by the finite element technique for fcc polycrystals. Materials Science and Engineering a-Structural Materials Properties Microstructure and Processing 257, 108-117.

Barlat, F., Maeda, Y., Chung, K., Yanagawa, M., Brem, J.C., Hayashida, Y., Lege, D.J., Matsui, K., Murtha, S.J., Hattori, S., Becker, R.C., Makosey, S., 1997. Yield function development for aluminum alloy sheets. Journal of the Mechanics and Physics of Solids 45, 1727-1763.

Becker, R., 1991. Analysis of texture evolution in channel die compression-I. Effects of grain interaction. Acta Metallurgica Et Materialia 39, 1211-1230.

Beyerlein, I.J., Tomé, C.N., 2007. Modeling transients in the mechanical response of copper due to strain path changes. International Journal of Plasticity 23, 640-664.

Bhattacharyya, A., El-Danaf, E., Kalidindi, S.R., Doherty, R.D., 2001. Evolution of grain-scale microstructure during large strain simple compression of polycrystalline aluminum with quasicolumnar grains: OIM measurements and numerical simulations. International Journal of Plasticity 17, 861-883.

Bron, F., Besson, J., 2004. A yield function for anisotropic materials Application to aluminum alloys. International Journal of Plasticity 20, 937-963.

Bronkhorst, C.A., Kalidindi, S.R., Anand, L., 1991. AN EXPERIMENTAL AND ANALYTICAL STUDY OF THE EVOLUTION OF CRYSTALLOGRAPHIC TEXTURING IN FCC MATERIALS. Textures and Microstructures 14, 1031-1036.

Bunge, H.-J., 1969. Texture analysis in materials science: mathematical methods. Elsevier. 
Chen, W., Pan, E., Wang, H., Zhang, C., 2010. Theory of indentation on multiferroic composite materials. Journal of the Mechanics and Physics of Solids 58, 1524-1551.

Dahlberg, C., Saito, Y., Öztop, M.S., Kysar, J.W., 2014. Geometrically necessary dislocation density measurements associated with different angles of indentations. International Journal of Plasticity 54, 81-95.

Dao, M., Chollacoop, N., Van Vliet, K.J., Venkatesh, T.A., Suresh, S., 2001. Computational modeling of the forward and reverse problems in instrumented sharp indentation. Acta Materialia 49, 3899-3918.

Delannay, L., Melchior, M.A., Signorelli, J.W., Remacle, J.F., Kuwabara, T., 2009. Influence of grain shape on the planar anisotropy of rolled steel sheets - evaluation of three models. Computational Materials Science 45, 739-743.

Doerner, M.F., Nix, W.D., 1986. A method for interpreting the data from depth-sensing indentation instruments. Journal of Materials Research 1, 601-609.

Donohue, B.R., Ambrus, A., Kalidindi, S.R., 2012. Critical evaluation of the indentation data analyses methods for the extraction of isotropic uniaxial mechanical properties using finite element models. Acta Materialia 60, 3943-3952.

Duchêne, L., El Houdaigui, F., Habraken, A.M., 2007. Length changes and texture prediction during free end torsion test of copper bars with FEM and remeshing techniques. International Journal of Plasticity 23, 1417-1438.

Fullwood, D.T., Niezgoda, S.R., Adams, B.L., Kalidindi, S.R., 2010. Microstructure sensitive design for performance optimization. Progress in Materials Science 55, 477-562.

Gambin, W., Barlat, F., 1997. Modeling of deformation texture development based on rate independent crystal plasticity. International Journal of Plasticity 13, 75-85.

Gao, Y.F., Pharr, G.M., 2007. Multidimensional contact moduli of elastically anisotropic solids. Scripta Materialia 57, 13-16.

Hannula, S.-P., Stone, D., Li, C.-Y., 1985. DETERMINATION OF TIME-DEPENDENT PLASTIC PROPERTIES OF METALS BY INDENTATION LOAD RELAXATION TECHNIQUES, Materials Research Society Symposia Proceedings, pp. 217-224.

Hertz, 1896. Miscellaneous Papers. MacMillan and Co. Ltd.,.

Hill, R., 1948. A Theory of the Yielding and Plastic Flow of Anisotropic Metals. Proceedings of the Royal Society of London A: Mathematical, Physical and Engineering Sciences 193, 281-297. 
Hull, D., 1963. Orientation and Temperature Dependence of Plastic Deformation Processes in $3.25 \%$ Silicon Iron. Proceedings of the Royal Society of London A: Mathematical, Physical and Engineering Sciences 274, 5-20.

Kalidindi, S.R., Anand, L., 1992. AN APPROXIMATE PROCEDURE FOR PREDICTING THE EVOLUTION OF CRYSTALLOGRAPHIC TEXTURE IN BULK DEFORMATION PROCESSING OF FCC METALS. International Journal of Mechanical Sciences 34, 309-329.

Kalidindi, S.R., Anand, L., 1993. LARGE DEFORMATION SIMPLE COMPRESSION OF A COPPER SINGLE-CRYSTAL. Metallurgical Transactions a-Physical Metallurgy and Materials Science 24, 989-992.

Kalidindi, S.R., Anand, L., 1994. MACROSCOPIC SHAPE CHANGE AND EVOLUTION OF CRYSTALLOGRAPHIC TEXTURE IN PRE-TEXTURED FCC METALS. Journal of the Mechanics and Physics of Solids 42, 459-490.

Kalidindi, S.R., Bhattacharya, A., Doherty, R., 2004. Detailed Analysis of Plastic Deformation in Columnar Polycrystalline Aluminum Using Orientation Image Mapping and Crystal Plasticity Models. Proceedings of the Royal Society of London: Mathematical, Physical and Engineering Sciences. 460, 1935 - 1956

Kalidindi, S.R., Bronkhorst, C.A., Anand, L., 1992. CRYSTALLOGRAPHIC TEXTURE EVOLUTION IN BULK DEFORMATION PROCESSING OF FCC METALS. Journal of the Mechanics and Physics of Solids 40, 537-569.

Kalidindi, S.R., Donohue, B.R., Li, S.Y., 2009. Modeling texture evolution in equal channel angular extrusion using crystal plasticity finite element models. International Journal of Plasticity $25,768-779$.

Kalidindi, S.R., Pathak, S., 2008. Determination of the effective zero-point and the extraction of spherical nanoindentation stress-strain curves. Acta Materialia 56, 3523-3532.

Kalidindi, S.R., Schoenfeld, S.E., 2000. On the prediction of yield surfaces by the crystal plasticity models for fcc polycrystals. Materials Science and Engineering a-Structural Materials Properties Microstructure and Processing 293, 120-129.

Kalidindi, S.R., Vachhani, S.J., 2014. Mechanical characterization of grain boundaries using nanoindentation. Current Opinion in Solid State and Materials Science.

Klusemann, B., Svendsen, B., Vehoff, H., 2012. Investigation of the deformation behavior of $\mathrm{Fe}-3 \% \mathrm{Si}$ sheet metal with large grains via crystal plasticity and finite-element modeling. Computational Materials Science 52, 25-32.

Knezevic, M., Levinson, A., Harris, R., Mishra, R.K., Doherty, R.D., Kalidindi, S.R., 2010. Deformation twinning in AZ31: Influence on strain hardening and texture evolution. Acta Materialia 58, 6230-6242. 
Kocks, U.F., Tomé, C.N., Wenk, H.R., 2000. Texture and Anisotropy: Preferred Orientations in Polycrystals and Their Effect on Materials Properties. Cambridge University Press.

Kucharski, S., Mróz, Z., 2001. Identification of plastic hardening parameters of metals from spherical indentation tests. Materials Science and Engineering A 318, 65-76.

Lebensohn, R.A., Tomé, C.N., 1993. A self-consistent anisotropic approach for the simulation of plastic deformation and texture development of polycrystals: Application to zirconium alloys. Acta Metallurgica Et Materialia 41, 2611-2624.

Li, N., Mara, N.A., Wang, Y.Q., Nastasi, M., Misra, A., 2011. Compressive flow behavior of Cu thin films and $\mathrm{Cu} / \mathrm{Nb}$ multilayers containing nanometer-scale helium bubbles. Scripta Materialia 64, 974-977.

Liu, Y., Varghese, S., Ma, J., Yoshino, M., Lu, H., Komanduri, R., 2008. Orientation effects in nanoindentation of single crystal copper. International Journal of Plasticity 24, 1990-2015.

Mayo, M.J., Nix, W.D., 1988. A micro-indentation study of superplasticity in $\mathrm{Pb}, \mathrm{Sn}$, and $\mathrm{Sn}-38$ wt\% Pb. Acta Metallurgica 36, 2183-2192.

Nair, A.K., Parker, E., Gaudreau, P., Farkas, D., Kriz, R.D., 2008. Size effects in indentation response of thin films at the nanoscale: A molecular dynamics study. International Journal of Plasticity 24, 2016-2031.

Oliver, W.C., Pharr, G.M., 1992. An improved technique for determining hardness and elastic modulus using load and displacement sensing indentation experiments. Journal of Materials Research 7, 1564-1583.

Oliver, W.C., Pharr, G.M., 2004. Measurement of hardness and elastic modulus by instrumented indentation: Advances in understanding and refinements to methodology. Journal of materials research 19, 3-20.

Orlans-Joliet, B., Driver, J.H., Montheillet, F., 1990. Plane strain compression of silicon-iron single crystals. Acta Metallurgica et Materialia 38, 581-594.

Patel, D.K., Al-Harbi, H.F., Kalidindi, S.R., 2014. Extracting single-crystal elastic constants from polycrystalline samples using spherical nanoindentation and orientation measurements. Acta Materialia 79, 108-116.

Patel, D.K., Kalidindi, S.R., 2016. Correlation of spherical nanoindentation stress-strain curves to simple compression stress-strain curves for elastic-plastic isotropic materials using finite element models. Acta Materialia 112, 295-302.

Pathak, S., 2009. Development and validation of a novel data analysis procedure for spherical nanoindentation. Drexel University. 
Pathak, S., Kalidindi, S.R., 2015. Spherical nanoindentation stress-strain curves. Materials Science and Engineering: R: Reports 91, 1-36.

Pathak, S., Kalidindi, S.R., Klemenz, C., Orlovskaya, N., 2008. Analyzing indentation stressstrain response of $\mathrm{LaGaO} 3$ single crystals using spherical indenters. Journal of the European Ceramic Society 28, 2213-2220.

Pathak, S., Stojakovic, D., Kalidindi, S.R., 2009. Measurement of the local mechanical properties in polycrystalline samples using spherical nanoindentation and orientation imaging microscopy. Acta Materialia 57, 3020-3028.

Pethicai, J., Hutchings, R., Oliver, W.C., 1983. Hardness measurement at penetration depths as small as $20 \mathrm{~nm}$. Philosophical Magazine A 48, 593-606.

Pharr, G.M., 1998. Measurement of mechanical properties by ultra-low load indentation. Materials Science and Engineering A 253, 151-159.

Press, W.H., 2007. Numerical Recipes 3rd Edition: The Art of Scientific Computing. Cambridge University Press.

Proust, G., Kalidindi, S.R., 2006. Procedures for construction of anisotropic elastic-plastic property closures for face-centered cubic polycrystals using first-order bounding relations. Journal of the Mechanics and Physics of Solids 54, 1744-1762.

Sabnis, P., Mazière, M., Forest, S., Arakere, N.K., Ebrahimi, F., 2012. Effect of secondary orientation on notch-tip plasticity in superalloy single crystals. International Journal of Plasticity $28,102-123$.

Sabnis, P.A., Forest, S., Arakere, N.K., Yastrebov, V.A., 2013. Crystal plasticity analysis of cylindrical indentation on a Ni-base single crystal superalloy. International Journal of Plasticity 51, 200-217.

Sarma, G.B., Dawson, P.R., 1996. Texture predictions using a polycrystal plasticity model incorporating neighbor interactions. International Journal of Plasticity 12, 1023-1054.

Segurado, J., Lebensohn, R.A., Llorca, J., Tomé, C.N., 2012. Multiscale modeling of plasticity based on embedding the viscoplastic self-consistent formulation in implicit finite elements. International Journal of Plasticity 28, 124-140.

Shan, Z.W., Mishra, R.K., Syed Asif, S.A., Warren, O.L., Minor, A.M., 2008. Mechanical annealing and source-limited deformation in submicrometre-diameter Ni crystals. Nat Mater 7, 115-119.

Suits, J.C., Chalmers, B., 1961. Plastic microstrain in silicon-iron. Acta Metallurgica 9, 854-860. 
Swadener, J.G., Pharr, G.M., 2001. Indentation of elastically anisotropic half-spaces by cones and parabolae of revolution. Philos Mag A 81, 447-466.

Taylor, G.I., 1934. The Mechanism of Plastic Deformation of Crystals. Part I. Theoretical. Proceedings of the Royal Society of London A: Mathematical, Physical and Engineering Sciences 145, 362-387.

Taylor, G.I., 1938. Plastic strain in metals. 307-324.

Tikhovskiy, I., Raabe, D., Roters, F., 2007. Simulation of earing during deep drawing of an Al$3 \% \mathrm{Mg}$ alloy (AA 5754) using a texture component crystal plasticity FEM. Journal of Materials Processing Technology 183, 169-175.

Tomé, C.N., Lebensohn, R.A., Necker, C.T., 2002. Mechanical anisotropy and grain interaction in recrystallized aluminum. Metallurgical and Materials Transactions A: Physical Metallurgy and Materials Science 33, 2635-2648.

Tomé, C.N., Maudlin, P.J., Lebensohn, R.A., Kaschner, G.C., 2001. Mechanical response of zirconium-I. Derivation of a polycrystal constitutive law and finite element analysis. Acta Materialia 49, 3085-3096.

Uchic, M.D., Dimiduk, D.M., Florando, J.N., Nix, W.D., 2004. Sample Dimensions Influence Strength and Crystal Plasticity. Science 305, 986-989.

Vachhani, S.J., Doherty, R.D., Kalidindi, S.R., 2016. Studies of grain boundary regions in deformed polycrystalline aluminum using spherical nanoindentation. International Journal of Plasticity 81, 87-101.

Vachhani, S.J., Kalidindi, S.R., 2015. Grain-scale measurement of slip resistances in aluminum polycrystals using spherical nanoindentation. Acta Materialia 90, 27-36.

Vlassak, J.J., Ciavarella, M., Barber, J.R., Wang, X., 2003. The indentation modulus of elastically anisotropic materials for indenters of arbitrary shape. Journal of the Mechanics and Physics of Solids 51, 1701-1721.

Vlassak, J.J., Nix, W.D., 1993. Indentation Modulus of Elastically Anisotropic Half-Spaces. Philos Mag A 67, 1045-1056.

Vlassak, J.J., Nix, W.D., 1994. Measuring the elastic properties of anisotropic materials by means of indentation experiments. Journal of the Mechanics and Physics of Solids 42, 12231245.

Willis, J.R., 1966. Hertzian Contact of Anisotropic Bodies. Journal of the Mechanics and Physics of Solids 14, 163-\&. 
Yabansu, Y.C., Kalidindi, S.R., 2015. Representation and calibration of elastic localization kernels for a broad class of cubic polycrystals. Acta Materialia 94, 26-35.

Yabansu, Y.C., Patel, D.K., Kalidindi, S.R., 2014. Calibrated localization relationships for elastic response of polycrystalline aggregates. Acta Materialia 81, 151-160.

Zaafarani, N., Raabe, D., Singh, R.N., Roters, F., Zaefferer, S., 2006. Three-dimensional investigation of the texture and microstructure below a nanoindent in a $\mathrm{Cu}$ single crystal using 3D EBSD and crystal plasticity finite element simulations. Acta Materialia 54, 1863-1876.

Zbib, A.A., Bahr, D.F., 2007. Dislocation Nucleation and Source Activation during Nanoindentation Yield Points. Metallurgical and Materials Transactions A 38, 2249-2255. 